\title{
Growth impairment in undernourished children
}

The impaired growth phenotype of undernourished children can be transmitted to mice via the gut microbiota, according to new research.

The researchers took faecal samples from 19 Malawian children (nine were 6 months old and 10 were 18 months old) who either had a healthy or an undernourished growth phenotype. The samples were transplanted into 5 -week-old,

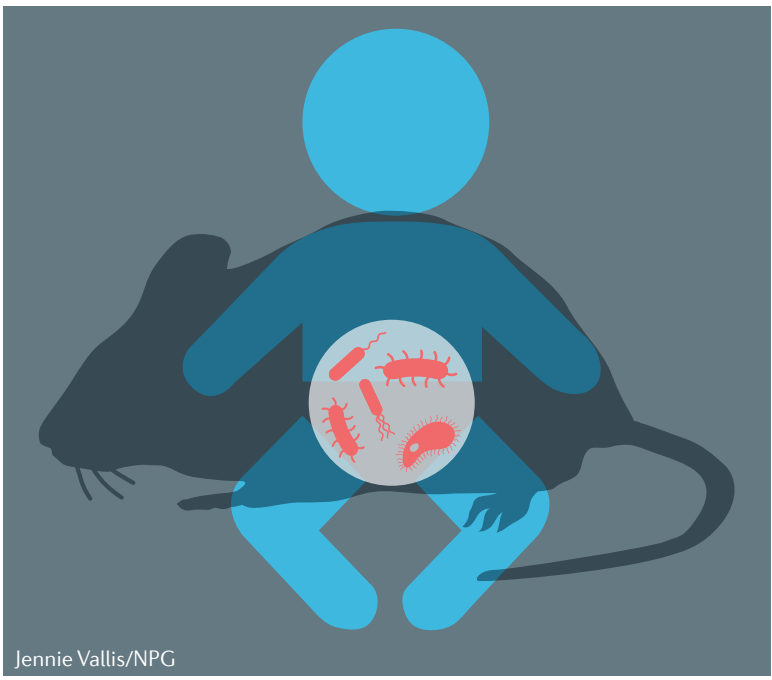

actively growing, germ-free C57BL/6J mice that were fed a Malawian diet before and after receiving the transplant. Over an observation period of 4-5 weeks, mice transplanted with microbiota from the healthy children gained more weight and lean body mass than mice that received microbiota from the undernourished children, despite similar levels of food consumption between the two groups. The microbiota from the 6-monthold children had greater effects on growth than the microbiota from the 18-month-old children.

Interestingly, when mice were cohoused shortly after receiving a faecal transplant from either an undernourished or a healthy child, the healthy microbiota was transmitted to the mice transplanted with a sample from the undernourished child and growth impairment was prevented. The growth abnormalities could also be prevented if Ruminococcus gnavus and Clostridium symbiosum were added to the microbiota samples of undernourished children before being transplanted into the mice.
The gut microbiota of children who are undernourished is usually immature, that is, more similar to microbiota of younger children than to that of age-matched healthy controls. "These findings provide evidence that microbiota immaturity is causally related to undernutrition," write the researchers.

In the paper, the investigators acknowledge that more work needs to be done, including undertaking similar analyses using microbiota from children in different geographical locations and with different cultural backgrounds, as well as investigating the effects of interventions to progress the maturity of the gut microbiota. This future work could have implications for designing interventions to effectively repair or prevent growth impairment and other abnormalities in undernourished children.

Claire Greenhill

ORIGINAL ARTICLE Blanton, L. V. et al.

Gut bacteria that prevent growth impairments transmitted by microbiota from malnourished children. Science 351, aad3311 (2016) 